\title{
Motivos de inscripción, permanencia y satisfacción en un programa de actividad física de mantenimiento para mujeres mayores
}

\author{
Statement of Enrollment, Retention and Satisfaction in a \\ Physical Activity Program for Women Over Maintenance
}

\section{Motivos de inscrição, permanencia e satisfação em um programa de atividade física para mulheres maiores}

\author{
Alberto Blázquez Manzano y Sebastián Feu Molina
}

Universidad de Extremadura, España

\begin{abstract}
Resumen: Se realizó un estudio para conocer los motivos de inscripción y permanencia en las actividades físicas de mantenimiento, así como la satisfacción de las usuarias que participan en el Programa de Dinamización Deportiva en las mancomunidades de municipios de Extremadura. En el estudio participaron 1605 usuarias mayores de 64 ańos del programa de AF de mantenimiento, con una edad media de $71.19 \pm 4.74$ ańos. Se utilizó una metodología de encuesta con instrumento validado por expertos y con una fiabilidad adecuada $(\alpha=.80)$. Los resultados indican que como principal motivo de inscripción y de permanencia en la práctica figura la salud y que la falta de tiempo es el principal motivo de abandono, encontrándose con diferencias significativas según la edad. La variable precio influye en la satisfacción del número horas semanales, la intensidad de las sesiones y la adecuación de las instalaciones. Por último, se pudo determinar que el coste económico de la actividad influye en la posibilidad de abandono cuando este es derivado de la falta de atención del monitor, la falta de variedad de las actividades y la falta de mantenimiento de las instalaciones.

Palabras clave: Gimnasia mantenimiento, mayores, mujer, satisfacción, coste.

Abstract: We conducted a study to determine the reasons for enrollment and adherence to physical activity maintenance that develop within the Sport Dynamization Program associations of municipalities in Extremadura, and satisfaction with it in relation to the price variable. Participants 1605 users aged 65 or physical activity program for maintenance. We conclude that health is the main reason for enrollment and adherence and lack
\end{abstract}

of time as the main reason for abandonment with significant differences by age. There is also a variable influence of price on satisfaction, number of hours per week, intensity of the sessions, fitness facilities. Finally, this variable influences the likelihood of abandonment resulting from the attention of the monitor, a variety of activities and lack of maintenance of facilities. Key words: Gymnastics maintenance, older women, satisfaction, cost Resumo: Foi realizado um estudo para conhecer os motivos da inscrição e permanência nas atividades físicas, assim como a satisfação das participantes do Programa de Dinamização Esportiva das "mancomunidades" dos municípios de Extremadura. O estudo contou com a participação de 1605 usuárias maiores de 64 anos do programa de Atividade Física e de manutenção, com uma idade media de $71.19 \pm 4.74$ anos. Utilizou-se o método de questionários como instrumento validado por expertos e com uma fiabilidade adequada $(\alpha=80)$. Os resultados indicam que o principal motivo da inscriçấo e da permanência na pratica da atividade física é a saúde e que a falta de tempo é o principal motivo do abandono, encontramos diferencias significativas na idade. A variável do preço influi na satisfaçáo do número de horas semanais, na intensidade de lesões e na adequação das instalaçôes. Por último, podemos determinar que o custo econômico da atividade influa na possibilidade de abandono quando este é derivado da falta de atenção do monitor, na falta de variedade das atividades e na falta de manutenção das instalaçōes.

Palavras chave: Ginástica, maiores, mulher, satisfação, custo

\section{Introducción}

El concepto de gimnasia de mantenimiento hace referencia a aquella actividad físico-deportiva caracterizada por ejercicios con bajo impacto sin desplazamientos bruscos y rápidos; donde el trabajo de tensión muscular se complementa con la flexibilidad, coordinación o corrección postural (Moreno y Marín de Oliveira, 2003). Estos programas están orientados

Correspondencia:

Alberto Blázquez Manzano

Mail: alberto.blazquez@juntaextremadura.net a la mejora de la salud a través de un ejercicio más individualizado, seguro y motivante, que promueve una adherencia hacia la práctica (Meredith, 1988; Quenneville y Sidney, 1992; Mahoney, 1993; Morrow y Gill, 1995); y donde el componente expresivo y rítmico tales como música o juegos, hacen que tenga una buena atracción en el público femenino (Macías y Moya, 2002, Águila, Sicilia, Muyor y Orta, 2009).

La actividad física se ha relacionado positivamente con la calidad de vida y la autoeficacia a través de la mejora del estado de salud física y mental (McAuley, Konopack, Motl, Rosengren y Morris, 2006; White, Wojcicki y McAuley; 2009; 
Umstattd, Wilcox y Dowda, 2011), especialmente en adultos mayores (Paxton, Motl, Aylward y Nigg 2011).

De igual modo, se ha demostrado el papel socializador de la actividad física especialmente en adultos y mayores (Prevc y Tepic, 2009; Sterbova, Hrubá, Harvanova, Elfmark y Otipkova; 2009); así como una mediación significativa entre la pertenencia al grupo y la asistencia a la sesión (Annesi, Unruh, Marti, Nathan, Gorjala y Tennant, 2011).

Han sido muchos los modelos que integran la satisfacción y la calidad del servicio percibido (Oliver, 1993; Spreng y Mackoy, 1996), y al mismo tiempo han sido comparados con las intenciones de compra (Woodside, Frey y Daly, 1989; Cronin y Taylor, 1992; Taylor y Baker, 1994, Choi, Cho, Lee, Lee, Kim, 2002), la fidelización de los clientes (Fornell, 1996; Johnson y Gustavson, 2000) o la frecuencia de práctica (Martínez-Tur, Peiró y Ramos, 1995). Sin embargo, hay autores que seńalan que la calidad percibida de un servicio se refiere a la evaluación global del mismo, mientras que la satisfacción haría referencia a la evaluación del proceso de transacción específicamente (Bitner, 1990; Bolton y Drew, 1991, Boulding, Kalra, Staelin, y Zeithml, 1993).

Conocer el perfil poblacional, así como sus necesidades y demandas, resulta clave a la hora de lograr una mayor competitividad (Mármol, Orquín Castrillón y Sainz de Baranda, 2010). En cuanto al conocimiento de la opinión de clientes, algunas líneas de trabajo actuales se centran en crear y adaptar métodos de medida a casos específicos. En el caso de los centros deportivos, algunos de los modelos que se aplican toman en consideración dimensiones de calidad como: entorno, interacción y resultado (Calabuig, Molina y Nuñez, 2012).

La percepción del cliente sobre el precio de un servicio deportivo tiene gran influencia tanto en sus intenciones de compra como en su grado de satisfacción con lo que ha consumido, siendo importante tener en cuenta que las estrategias que reducen la percepción de precios injustos elevan el valor percibido del producto o servicio (Sen y Johnson, 1997)

No obstante, el valor de un servicio no sólo está determinado por su cuantía económica sino también por la precisión con la que los compradores perciben dicho valor, y por la importancia que otorgan a conseguir lo máximo posible con su dinero o esfuerzo (Nagel, 1995). Otros autores indican que las actitudes hacia el servicio deportivo no difieren en función del nivel de experiencia del cliente (Martínez y Martínez, 2009). El valor no sólo toma muchas formas, sino que proviene de muchas fuentes: utilidad de un producto/servicio, su calidad, la imagen asociada, su disponibilidad y el/los servicio/s que lo acompaña (Magretta, 2002).

\section{Variables que influyen en la satisfacción}

Moreno y Marín de Oliveira (2003) señalan que los usuarios de programas de actividad física (AF) tradicional como la gimnasia de mantenimiento, valoran más los motivos relacionados con la salud que los de fitness, no resultando significativas las diferencias entre el medio acuático y terrestre.

Algunos estudios han encontrado diferencias motivacionales en la práctica de AF en función del sexo (Ruíz, García y Díaz, 2007; Pavón y Moreno, 2008; Rodríguez-Romo, Boned-Pascual y Garrido-Muñoz, 2009), y la edad (Cabańas, Martínez y Del Riego, 2004; Ruíz et al., 2007). En cuanto a las relaciones sociales algunos trabajos constatan un incremento del interés por la AF por esta causa cuando aumenta la edad, mientras que otros estudios han encontrado que a los más jóvenes le interesa la AF por diversión y mejor sus relaciones sociales (García, Hernández, Ońa, Godoy y Rebollo, 2001).

El clima psicológico también influye en la motivación hacia la práctica de AF (Weiss y Ebbeck, 1996; Smith, 1999), siendo el grupo de iguales una de las variables que influye en las autopercepciones y en la autoestima.

Los aspectos demográficos parecen condicionar la realización de AF para el mantenimiento de la salud, en los entornos urbanos donde parece haber un mayor nivel de práctica de AF que en los entornos rurales (Moreno y Gutiérrez, 1998; Reis, et al., 2004; Martín, et al., 2005); aunque esto puede relacionarse con las condiciones labores, o aspectos socioeconómicos más que por condiciones de carencia real de zonas rurales (Tay, et al., 2004). También se ha constatado que un nivel social superior favorece una mayor práctica de AF que entornos sociales más desfavorecidos (Moreno y Gutiérrez, 1998).

El objetivo de la presente investigación es conocer el principal motivo de inscripción y de permanencia de las mujeres mayores de 64 ańos que participan en los programas de actividad físico deportiva de mantenimiento de las mancomunidades de Extremadura en poblaciones menores de 20.000 habitantes; así como describir cuál es la valoración de la satisfacción con dicho servicio y determinar la influencia de la edad número de habitantes de las localidades y el coste económico de la actividad.

\section{Método}

Se realizó un estudio cuantitativo y descriptivo mediante encuesta, de carácter transversal por su aplicación en un único momento temporal (Montero y León, 2007).

\section{Población y muestra}

El estudio se realizó en los programas municipales de actividades físicas de mantenimiento que se desarrollan dentro del Programa de Dinamización Deportiva en las mancomunidades de municipios de Extremadura. Estos programas son abiertos a todo el público y son desarrollados por dinami- 
zadores deportivos, cada uno de los cuales gestiona varias localidades mancomunadas. Las encuestas se realizaron en todas las localidades donde se desarrollan los programas de actividad física de mantenimiento mencionados, solicitando la participación a todas las usuarias y usuarios. Sólo se consiguieron 56 encuestas entre los hombres, el 3.1\% para el rango de edad estudiado, por lo que se decidió realizar el estudio centrando el mismo, en las mujeres participantes. Por tanto, se realizaron 1605 encuestas entre las usuarias mayores de 64 años de los programas de AF de mantenimiento. La participación fue voluntaria y la muestra arrojó un error de muestreo de $2.4 \%$ para un IC 95\%. Las participantes tenían una edad media de $71.19 \pm 4.74$ ańos de edad.

\section{Variables}

Las variables estudiadas se concretaron en las siguientes: principal motivo de inscripción, principal motivo de permanencia y de abandono de la práctica deportiva en función de la edad de las usuarias. Seguidamente se analizó la valoración de la satisfacción de las usuarias en tres dimensiones: características de la instalación (5 variables), organización de la actividad (3 variables) y desarrollo de la misma (4 variables). Estas variables fueron analizadas en función de la edad, número de habitantes de la localidad y coste mensual de la actividad.

\section{Instrumento}

En la primera parte del cuestionario se tomaron datos sociodemográficas (edad y localidad) y contextuales (años de permanencia en el programa, número de días, sesiones semanales y coste económico de la actividad) mientras que en la segunda se pidió a las usuarias que identificaran el principal motivo (Rodríguez-Romo, et al., 2009) por el que se inscribían y el principal motivo por el que permanecían en la actividad.

Para el diseño de la escala de satisfacción de las usuarias con el servicio deportivo, tercera parte, se tuvieron en cuenta tres dimensiones bien delimitadas en trabajos previos: la satisfacción con la instalación (Wicker, Breuer y Pawlowski, 2009), la organización de la actividad en su dimensión temporal (Nuviala, Tamayo, Iranzo y Falcón, 2008) y el desarrollo de la misma (Graupera, Martínez y Martín, 2003). Se utilizó una escala Likert de cinco pasos para las respuestas de cada ítem de las tres dimensiones: Satisfacción con la instalación (iluminación, limpieza, dimensiones del espacio, accesibilidad y ventilación), Organización de la Actividad (horario de la actividad, duración de la clase y número de horas semanales) y Desarrollo de la Actividad (motivación de las sesiones, intensidad de las sesiones, aprovechamiento del tiempo y material deportivo). También se diseńaron tres ítems para conocer los motivos que les llevarían a abandonar la actividad desde la perspectiva de la calidad del servicio. La escala obtuvo una fiabilidad adecuada $(\alpha=.80)$.

\section{Procedimiento}

Inicialmente se realizó un análisis de la literatura para diseñar los bloques dos y tres del cuestionario: valoración de los motivos de inscripción y permanencia así como la satisfacción de las usuarias con el programa. La escala de satisfacción con el servicio se construyó desde la óptica multidimensional dominante en los estudios de satisfacción en el ámbito deportivo (Morales, Hernández-Mendo y Blanco, 2005; Tsitskari, Tsiotras y Tsiotras, 2006). Antes de pasar el instrumento a la muestra se pidió a un grupo de cinco expertos en la gestión del Programa de Dinamización Deportiva, la evaluación del cuestionario y sus aportaciones. Estos expertos eran licenciados y/o doctores en Ciencias de la actividad física y el deporte que trabajaban en el ámbito de la gestión deportiva.

Los cuestionarios en formato papel fueron pasados antes del inicio de las sesiones de prácticas por técnicos del programa de dinamización deportiva, los cuales fueron instruidos por el equipo investigador sobre el procedimiento de aplicación y tomando como criterio de inclusión todos aquellos usuarios que quisieran participar. Por tanto, la participación se le solicitó al $100 \%$ de la muestra que asistió a la práctica en el día del registro de los datos.

\section{Análisis estadístico}

Se realizó un análisis descriptivo básico de la muestra y un análisis de las variables nominales a través de tablas de contingencia analizando el grado de asociación de las variables a través del coeficiente de contingencia $(C)$; así como el grado de relación entre cada uno de los niveles de la variable a través de los residuos tipificados corregidos $(R T C)>1.96$ o $<-1.96$ (95\% de nivel de confianza).

También se desarrolló un análisis descriptivo de los ítems y posteriormente se buscaron diferencias significativas en la opinión de las usuarias en función del rango de edad y de la población. Para el análisis correlacional se analizaron los supuestos para elegir el tipo de prueba determinando que lo más idóneo eran pruebas no paramétricas. El análisis estadístico se realizó con el software SPSS Statistics 17.0.

\section{Resultados}

\section{Descriptivos de la muestra}

La edad media de todas las participantes fue de $71.19 \pm 4.74$ ańos. En la tabla 1 se puede observar que el menor grupo de edad es el de mayores de 79 años (6.1\%) y que el grupo que más acude a estas actividades es el de 65 a 69 años (42.7\%), 
seguido de las de 70 a 74 años (33.6\%). El mayor número de participantes reside en localidades entre 1000 y 5000 ha- bitantes, un $51.7 \%$, seguido de las localidades de menos de 1000 habitantes, un 41\%. (Tabla 1).

Tabla 1. Distribución de las participantes por edad y población de su lugar residencia

\begin{tabular}{|c|c|c|c|c|c|c|}
\hline \multirow{2}{*}{ Edades } & & \multicolumn{4}{|c|}{ Rango de habitantes } & \multirow[b]{2}{*}{ Total } \\
\hline & & $\begin{array}{c}\text { Menos de } 1000 \\
\text { hab. }\end{array}$ & $\begin{array}{c}\text { De } 1000 \text { a } 4999 \\
\text { hab. }\end{array}$ & $\begin{array}{c}\text { De } 5000 \text { a } 9999 \\
\text { hab. }\end{array}$ & $\begin{array}{l}\text { De } 10000 \text { a } \\
20000 \text { hab. }\end{array}$ & \\
\hline \multirow[t]{4}{*}{ 65-69 años } & Recuento & 284 & 342 & 47 & 13 & 686 \\
\hline & $\%$ dentro de edad & $41.4 \%$ & $49.9 \%$ & $6.9 \%$ & $1.9 \%$ & $100.0 \%$ \\
\hline & $\%$ dentro de Rango de habitantes & $43.2 \%$ & $41.3 \%$ & $46.5 \%$ & $76.5 \%$ & $42.7 \%$ \\
\hline & $\%$ del total & $17.7 \%$ & $21.3 \%$ & $2.9 \%$ & $.8 \%$ & $42.7 \%$ \\
\hline \multirow[t]{4}{*}{ 70-74 ańos } & Recuento & 217 & 291 & 30 & 2 & 540 \\
\hline & $\%$ dentro de edad & $40.2 \%$ & $53.9 \%$ & $5.6 \%$ & $.4 \%$ & $100.0 \%$ \\
\hline & $\%$ dentro de Rango de habitantes & $33.0 \%$ & $35.1 \%$ & $29.7 \%$ & $11.8 \%$ & $33.6 \%$ \\
\hline & $\%$ del total & $13.5 \%$ & $18.1 \%$ & $1.9 \%$ & $.1 \%$ & $33.6 \%$ \\
\hline \multirow[t]{4}{*}{ 75-79 años } & Recuento & 126 & 138 & 16 & 1 & 281 \\
\hline & $\%$ dentro de edad & $44.8 \%$ & $49.1 \%$ & $5.7 \%$ & $.4 \%$ & $100.0 \%$ \\
\hline & $\%$ dentro de Rango de habitantes & $19.1 \%$ & $16.6 \%$ & $15.8 \%$ & $5.9 \%$ & $17.5 \%$ \\
\hline & $\%$ del total & $7.9 \%$ & $8.6 \%$ & $1.0 \%$ & $.1 \%$ & $17.5 \%$ \\
\hline \multirow[t]{4}{*}{ + 79 años } & Recuento & 31 & 58 & 8 & 1 & 98 \\
\hline & $\%$ dentro de edad & $31.6 \%$ & $59.2 \%$ & $8.2 \%$ & $1.0 \%$ & $100.0 \%$ \\
\hline & $\%$ dentro de Rango de habitantes & $4.7 \%$ & $7.0 \%$ & $7.9 \%$ & $5.9 \%$ & $6.1 \%$ \\
\hline & $\%$ del total & $1.9 \%$ & $3.6 \%$ & $.5 \%$ & $.1 \%$ & $6.1 \%$ \\
\hline \multirow[t]{4}{*}{ Total } & Recuento & 658 & 829 & 101 & 17 & 1605 \\
\hline & $\%$ dentro de edad & $41.0 \%$ & $51.7 \%$ & $6.3 \%$ & $1.1 \%$ & $100.0 \%$ \\
\hline & $\%$ dentro de Rango de habitantes & $100.0 \%$ & $100.0 \%$ & $100.0 \%$ & $100.0 \%$ & $100.0 \%$ \\
\hline & $\%$ del total & $41.0 \%$ & $51.7 \%$ & $6.3 \%$ & $1.1 \%$ & $100.0 \%$ \\
\hline
\end{tabular}

Las mujeres participan en el programa de gimnasia de mantenimiento una media de $2.35 \pm .58$ días a la semana. El $68 \%$ asiste dos días en semana de AF de mantenimiento, mientras que el $28.2 \%$ asiste tres días, el $2.1 \%$ cuatro días, el $1 \%$ asiste cinco días y sólo un $0.6 \%$ asiste un día a la actividad. Las usuarias realizan una media de $2.69 \pm 1.10$ horas de práctica la semana. En cuanto a la continuidad, el 33.5\% lleva más de seis años en el programa de AF de mantenimiento desarrollado a través del Programa de Dinamización Deportiva de Extremadura, el $31.4 \%$ lleva de uno a tres ańos, el $18.4 \%$ de cuatro a seis ańos y el $16.7 \%$ menos de un año.

\section{Relación entre los motivos de inscripción y permanencia en el programa de AF de mantenimiento}

Se estudió la asociación entre los motivos de inscripción al programa de AF de mantenimiento y los motivos de permanencia al mismo. Los resultados de Chi-cuadrado muestran una alta asociación entre el principal motivo de inscripción en la AF de mantenimiento y el principal motivo de perma- nencia a la misma $(X 2(\mathrm{gl}=16)=1825.70 ; p<.01)$. A través del coeficiente de contingencia se comprobó que existía una alta asociación entre las variables $(C=.730 ; p<.01)$.

El análisis de los descriptivos y los RTC de la tabla de contingencia mostró la existencia de tendencias y asociaciones entre los motivos de inscripción y permanencia (Tabla 2). El principal motivo de inscripción en las AF de mantenimiento fue la salud (84.8\%), seguido de la recreación (5.7\%), relacionarse con gente $(4.8 \%)$ y la preparación para hacer deporte (3.1\%). La estética ocupó el último lugar como motivo para inscribirse en la AF de mantenimiento (1.6\%). Por otro lado, el principal motivo para adherirse al programa, es la salud (85.8\%), aumentando el porcentaje de las que se inscribieron por este motivo. El segundo motivo es relacionarse con gente (5.9\%) seguido de la recreación (5.7\%). Solo el 1,6\% decide inscribirse y mantenerse por preparación deportiva.

Estos datos se ven confirmados por los RTC indicando que hay muchos más casos de los esperados en la salud como motivo de inscripción y permanencia en la AF de mantenimiento $(R T C>1.96)$. 
Tabla 2. Tabla de contingencia entre los motivos de inscripción y permanencia a la AF de mantenimiento

\begin{tabular}{|c|c|c|c|c|c|c|c|}
\hline \multirow[b]{2}{*}{$\begin{array}{l}\text { Motivo de } \\
\text { inscripción }\end{array}$} & & \multicolumn{5}{|c|}{ Motivos de permanencia en la práctica } & \multirow[b]{2}{*}{ Total } \\
\hline & & Estética & Salud & Recreación & $\begin{array}{c}\text { Relacionarse } \\
\text { con gente }\end{array}$ & $\begin{array}{c}\text { Preparación para el } \\
\text { deporte }\end{array}$ & \\
\hline \multirow{5}{*}{ Estética } & Recuento & 8 & 17 & 1 & 0 & 0 & 26 \\
\hline & \% Motivo de inscripción & $30.8 \%$ & $65.4 \%$ & $3.8 \%$ & $.0 \%$ & $.0 \%$ & $100.0 \%$ \\
\hline & $\%$ Motivos permanencia & $53.3 \%$ & $1.2 \%$ & $1.1 \%$ & $.0 \%$ & $.0 \%$ & $1.6 \%$ \\
\hline & $\%$ del total & $.5 \%$ & $1.1 \%$ & $.1 \%$ & $.0 \%$ & $.0 \%$ & $1.6 \%$ \\
\hline & RTC & 15.9 & -3.0 & -.4 & -1.3 & -.6 & \\
\hline \multirow{5}{*}{ Salud } & Recuento & 6 & 1283 & 25 & 38 & 5 & 1357 \\
\hline & \% Motivo de inscripción & $.4 \%$ & $94.5 \%$ & $1.8 \%$ & $2.8 \%$ & $.4 \%$ & $100.0 \%$ \\
\hline & \% Motivos permanencia & $40.0 \%$ & $93.4 \%$ & $27.5 \%$ & $39.6 \%$ & $20.0 \%$ & $84.8 \%$ \\
\hline & $\%$ del total & $.4 \%$ & $80.1 \%$ & $1.6 \%$ & $2.4 \%$ & $.3 \%$ & $84.8 \%$ \\
\hline & RTC & -4.8 & 23.6 & -15.7 & -12.7 & -9.1 & \\
\hline \multirow{5}{*}{ Recreación } & Recuento & 0 & 24 & 60 & 8 & 0 & 92 \\
\hline & \% Motivo de inscripción & $.0 \%$ & $26.1 \%$ & $65.2 \%$ & $8.7 \%$ & $.0 \%$ & $100.0 \%$ \\
\hline & \% Motivos permanencia & $.0 \%$ & $1.7 \%$ & $65.9 \%$ & $8.3 \%$ & $.0 \%$ & $5.7 \%$ \\
\hline & $\%$ del total & $.0 \%$ & $1.5 \%$ & $3.7 \%$ & $.5 \%$ & $.0 \%$ & $5.7 \%$ \\
\hline & RTC & -1.0 & -16.9 & 25.4 & 1.1 & -1.2 & \\
\hline \multirow{5}{*}{$\begin{array}{l}\text { Relacionarse } \\
\text { con gente }\end{array}$} & Recuento & 0 & 23 & 2 & 50 & 2 & 77 \\
\hline & \% Motivo de inscripción & $.0 \%$ & $29.9 \%$ & $2.6 \%$ & $64.9 \%$ & $2.6 \%$ & $100.0 \%$ \\
\hline & $\%$ Motivos permanencia & $.0 \%$ & $1.7 \%$ & $2.2 \%$ & $52.1 \%$ & $8.0 \%$ & $4.8 \%$ \\
\hline & $\%$ del total & $.0 \%$ & $1.4 \%$ & $.1 \%$ & $3.1 \%$ & $.1 \%$ & $4.8 \%$ \\
\hline & RTC & -.9 & -14.4 & -1.2 & 22.3 & .8 & \\
\hline \multirow{5}{*}{$\begin{array}{l}\text { Preparación } \\
\text { para el deporte }\end{array}$} & Recuento & 1 & 27 & 3 & 0 & 18 & 49 \\
\hline & \% Motivo de inscripción & $2.0 \%$ & $55.1 \%$ & $6.1 \%$ & $.0 \%$ & $36.7 \%$ & $100.0 \%$ \\
\hline & $\%$ Motivos permanencia & $6.7 \%$ & $2.0 \%$ & $3.3 \%$ & $.0 \%$ & $72.0 \%$ & $3.1 \%$ \\
\hline & $\%$ del total & $.1 \%$ & $1.7 \%$ & $.2 \%$ & $.0 \%$ & $1.1 \%$ & $3.1 \%$ \\
\hline & $R T C$ & .8 & -6.3 & .1 & -1.8 & 20.2 & \\
\hline \multirow{4}{*}{ Total } & Recuento & 15 & 1374 & 91 & 96 & 25 & 1601 \\
\hline & \% Motivo de inscripción & $.9 \%$ & $85.8 \%$ & $5.7 \%$ & $6.0 \%$ & $1.6 \%$ & $100.0 \%$ \\
\hline & $\%$ Motivos permanencia & $100.0 \%$ & $100.0 \%$ & $100.0 \%$ & $100.0 \%$ & $100.0 \%$ & $100.0 \%$ \\
\hline & $\%$ del total & $.9 \%$ & $85.8 \%$ & $5.7 \%$ & $6.0 \%$ & $1.6 \%$ & $100.0 \%$ \\
\hline
\end{tabular}

Relación entre los motivos de inscripción y la edad

Los resultados de Chi-cuadrado $(X 2(\mathrm{gl}=12)=34.48 ; p<.01)$ indican que hay asociación entre las variables, aunque valorado el coeficiente de contingencia se comprobó que la asociación era baja $(C=.147)$. A pesar de que la asociación es baja, del análisis de los $R T C$ se desprende que en todos los sectores de edad valoran la salud como principal motivo de inscripción $(R T C<|.196|)$ con un porcentaje superior al 75\% para cada sector de edad (tabla 3 ). En las mujeres con edades entre 65 y 69 años hay más casos de los esperados en donde se explicita como principal motivo para la inscripción en un programa de AF preparación para hacer deporte $(R T C>1.96)$, y menos para relacionarse con los demás $(R T C<-1.96)$. Entre los 70 y 74 años hay más casos de los esperados de personas que se inscriben por relación social (Tabla 3). 
Tabla 3. Motivos de inscripción en un programa de AF de mantenimiento en función de la edad

\begin{tabular}{|c|c|c|c|c|c|c|}
\hline \multirow{2}{*}{ Motivos de inscripción } & & \multicolumn{4}{|c|}{ Edades } & \multirow[t]{2}{*}{ Total } \\
\hline & & 65-69 años & 70-74 años & 75-79 años & +79 años & \\
\hline \multirow[t]{5}{*}{ Estética } & Recuento & 13 & 7 & 5 & 1 & 26 \\
\hline & \% Motivo de inscripción & $50.0 \%$ & $26.9 \%$ & $19.2 \%$ & $3.8 \%$ & $100.0 \%$ \\
\hline & $\%$ dentro de Edades & $1.9 \%$ & $1.3 \%$ & $1.8 \%$ & $1.0 \%$ & $1.6 \%$ \\
\hline & $\%$ del total & $.8 \%$ & $.4 \%$ & $.3 \%$ & $.1 \%$ & $1.6 \%$ \\
\hline & $R T C$ & .8 & -.7 & .2 & -.5 & \\
\hline \multirow[t]{5}{*}{ Salud } & Recuento & 591 & 452 & 244 & 74 & 1361 \\
\hline & \% Motivo de inscripción & $43.4 \%$ & $33.2 \%$ & $17.9 \%$ & $5.4 \%$ & $100.0 \%$ \\
\hline & $\%$ dentro de Edades & $86.2 \%$ & $83.7 \%$ & $86.8 \%$ & $75.5 \%$ & $84.8 \%$ \\
\hline & $\%$ del total & $36.8 \%$ & $28.2 \%$ & $15.2 \%$ & $4.6 \%$ & $84.8 \%$ \\
\hline & RTC & 1.3 & -.9 & 1.0 & -2.6 & \\
\hline \multirow[t]{5}{*}{ Recreación } & Recuento & 33 & 30 & 14 & 15 & 92 \\
\hline & \% Motivo de inscripción & $35.9 \%$ & $32.6 \%$ & $15.2 \%$ & $16.3 \%$ & $100.0 \%$ \\
\hline & $\%$ dentro de Edades & $4.8 \%$ & $5.6 \%$ & $5.0 \%$ & $15.3 \%$ & $5.7 \%$ \\
\hline & $\%$ del total & $2.1 \%$ & $1.9 \%$ & $.9 \%$ & $.9 \%$ & $5.7 \%$ \\
\hline & RTC & -1.4 & -.2 & -.6 & 4.2 & \\
\hline \multirow[t]{5}{*}{ Relacionarse con gente } & Recuento & 21 & 37 & 12 & 7 & 77 \\
\hline & \% Motivo de inscripción & $27.3 \%$ & $48.1 \%$ & $15.6 \%$ & $9.1 \%$ & $100.0 \%$ \\
\hline & $\%$ dentro de Edades & $3.1 \%$ & $6.9 \%$ & $4.3 \%$ & $7.1 \%$ & $4.8 \%$ \\
\hline & $\%$ del total & $1.3 \%$ & $2.3 \%$ & $.7 \%$ & $.4 \%$ & $4.8 \%$ \\
\hline & RTC & -2.8 & 2.7 & -.5 & 1.1 & \\
\hline \multirow[t]{5}{*}{ Preparación para el deporte } & Recuento & 28 & 14 & 6 & 1 & 49 \\
\hline & \% Motivo de inscripción & $57.1 \%$ & $28.6 \%$ & $12.2 \%$ & $2.0 \%$ & $100.0 \%$ \\
\hline & $\%$ dentro de Edades & $4.1 \%$ & $2.6 \%$ & $2.1 \%$ & $1.0 \%$ & $3.1 \%$ \\
\hline & $\%$ del total & $1.7 \%$ & $.9 \%$ & $.4 \%$ & $.1 \%$ & $3.1 \%$ \\
\hline & $R T C$ & 2.1 & -.8 & -1.0 & -1.2 & \\
\hline \multirow[t]{4}{*}{ Total } & Recuento & 686 & 540 & 281 & 98 & 1605 \\
\hline & \% Motivo de inscripción & $42.7 \%$ & $33.6 \%$ & $17.5 \%$ & $6.1 \%$ & $100.0 \%$ \\
\hline & $\%$ dentro de Edades & $100.0 \%$ & $100.0 \%$ & $100.0 \%$ & $100.0 \%$ & $100.0 \%$ \\
\hline & $\%$ del total & $42.7 \%$ & $33.6 \%$ & $17.5 \%$ & $6.1 \%$ & $100.0 \%$ \\
\hline
\end{tabular}

Relación entre los motivos de permanencia en la AF y la edad

A través de una tabla de contingencia se estudió la asociación entre los motivos de permanencia, variable nominal, y la edad de las usuarias de programas de AF mantenimiento. Más del $75 \%$ de las mujeres de cada sector de edad valoran la salud como principal motivo para mantenerse en la práctica de AF de mantenimiento.

Los resultados de Chi-cuadrado indican que no hay aso- ciación entre las variables $(X 2(\mathrm{gl}=12)=18.23 ; p>.05)$. El coeficiente de contingencia confirma que la asociación entre las variables es muy baja $(C=.106)$. Aún siendo la asociación es baja, el análisis de los RTC informa que a partir de los 79 años hay menos casos de los esperados que valoran la salud como principal motivo para mantenerse en un programa de AF de mantenimiento (RTC >-.196) y más casos de los esperados que valoran la recreación $(R T C>196)$, (Tabla 4). 
Tabla 4. Motivos de permanencia en un programa de AF de mantenimiento en función de la edad

\begin{tabular}{|c|c|c|c|c|c|c|}
\hline \multirow[b]{2}{*}{ Motivos de inscripción } & & \multicolumn{4}{|c|}{ Edades } & \multirow[t]{2}{*}{ Total } \\
\hline & & 65-69 años & 70-74 años & 75-79 años & +79 años & \\
\hline \multirow[t]{5}{*}{ Estética } & Recuento & 7 & 4 & 3 & 1 & 15 \\
\hline & $\%$ Motivo de permanencia & $46.7 \%$ & $26.7 \%$ & $20.0 \%$ & $6.7 \%$ & $100.0 \%$ \\
\hline & $\%$ dentro de Edades & $1.0 \%$ & $.7 \%$ & $1.1 \%$ & $1.0 \%$ & $.9 \%$ \\
\hline & $\%$ del total & $.4 \%$ & $.2 \%$ & $.2 \%$ & $.1 \%$ & $.9 \%$ \\
\hline & RTC & .3 & -.6 & .3 & .1 & \\
\hline \multirow[t]{5}{*}{ Salud } & Recuento & 591 & 465 & 243 & 75 & 1374 \\
\hline & $\%$ Motivo de permanencia & $43.0 \%$ & $33.8 \%$ & $17.7 \%$ & $5.5 \%$ & $100.0 \%$ \\
\hline & $\%$ dentro de Edades & $86.3 \%$ & $86.1 \%$ & $87.1 \%$ & $77.3 \%$ & $85.8 \%$ \\
\hline & $\%$ del total & $36.9 \%$ & $29.0 \%$ & $15.2 \%$ & $4.7 \%$ & $85.8 \%$ \\
\hline & RTC & .5 & .2 & .7 & -2.5 & \\
\hline \multirow[t]{5}{*}{ Recreación } & Recuento & 37 & 26 & 14 & 14 & 91 \\
\hline & $\%$ Motivo de permanencia & $40.7 \%$ & $28.6 \%$ & $15.4 \%$ & $15.4 \%$ & $100.0 \%$ \\
\hline & $\%$ dentro de Edades & $5.4 \%$ & $4.8 \%$ & $5.0 \%$ & $14.4 \%$ & $5.7 \%$ \\
\hline & $\%$ del total & $2.3 \%$ & $1.6 \%$ & $.9 \%$ & $.9 \%$ & $5.7 \%$ \\
\hline & $R T C$ & -.4 & -1.1 & -.5 & 3.8 & \\
\hline \multirow[t]{5}{*}{ Relacionarse con gente } & Recuento & 40 & 34 & 15 & 7 & 96 \\
\hline & $\%$ Motivo de permanencia & $41.7 \%$ & $35.4 \%$ & $15.6 \%$ & $7.3 \%$ & $100.0 \%$ \\
\hline & $\%$ dentro de Edades & $5.8 \%$ & $6.3 \%$ & $5.4 \%$ & $7.2 \%$ & $6.0 \%$ \\
\hline & $\%$ del total & $2.5 \%$ & $2.1 \%$ & $.9 \%$ & $.4 \%$ & $6.0 \%$ \\
\hline & RTC & -.2 & .4 & -.5 & .5 & \\
\hline \multirow{5}{*}{$\begin{array}{l}\text { Preparación para el } \\
\text { deporte }\end{array}$} & Recuento & 10 & 11 & 4 & 0 & 25 \\
\hline & $\%$ Motivo de permanencia & $40.0 \%$ & $44.0 \%$ & $16.0 \%$ & $.0 \%$ & $100.0 \%$ \\
\hline & $\%$ dentro de Edades & $1.5 \%$ & $2.0 \%$ & $1.4 \%$ & $.0 \%$ & $1.6 \%$ \\
\hline & $\%$ del total & $.6 \%$ & $.7 \%$ & $.2 \%$ & $.0 \%$ & $1.6 \%$ \\
\hline & $R T C$ & -.3 & 1.1 & -.2 & -1.3 & \\
\hline \multirow[t]{4}{*}{ Total } & Recuento & 685 & 540 & 279 & 97 & 1601 \\
\hline & $\%$ Motivo de permanencia & $42.8 \%$ & $33.7 \%$ & $17.4 \%$ & $6.1 \%$ & $100.0 \%$ \\
\hline & $\%$ dentro de Edades & $100.0 \%$ & $100.0 \%$ & $100.0 \%$ & $100.0 \%$ & $100.0 \%$ \\
\hline & $\%$ del total & $42.8 \%$ & $33.7 \%$ & $17.4 \%$ & $6.1 \%$ & $100.0 \%$ \\
\hline
\end{tabular}

Por otro lado, a través de una escala de cinco pasos $(1-5)$, se les preguntó a las usuarias por la mejora que han percibido en el plano físico, psíquico y social. Los datos de la media indican una percepción de mejora especialmente en el aspecto psíquico $(M=4.66 \pm .59)$, seguido de cerca del físico $(M=4.57 \pm .64)$ y social $(M=4.58 \pm .73)$. No se encontraron diferencias significativas en la percepción según los rangos de edad.

\section{Satisfacción de las usuarias}

La satisfacción de las usuarias por la actividad fue de satisfechas a muy satisfechas. A través de la prueba $h$ de Kruskal Wallis se buscaron diferencias significativas en las variables de satisfacción estudiadas en función de los habitantes de la localidad y de los rangos de edad de las participantes, Tabla 5.

Las usuarias del programa de AF de mantenimiento valoran satisfactoriamente casi todos los ítems evaluados, siendo la puntuación en su mayoría por encima de 4 (Tabla 5). En el caso de las variables limpieza, dimensiones del lugar accesibilidad, ventilación, número de horas semanales y adecuación del material deportivo la desviación típica es de más de un punto. La valoración de la adecuación de las instalaciones deportivas es regular $3.60 \pm 1.21$.

Se encontraron diferencias significativas en función del número de habitantes en la satisfacción con la iluminación, limpieza, dimensiones del lugar, accesibilidad, ventilación horario de la actividad, duración y aprovechamiento de la clase $(p<.01)$; además de la intensidad de las sesiones $(p<.01)$. O dicho de otra forma, el número de habitantes es una variable que no encuentra diferencias significativas en el número de horas semanales, motivación de las sesiones, adecuación del material e instalaciones. También se encontraron diferencias significativas en función de los rangos de edad respecto a la ventilación $(p<.05)$ e iluminación $(p<.01)$. 
Tabla 5. Descriptivos de los ítems para conocer la satisfacción de las usuarias

\begin{tabular}{|c|c|c|c|c|c|c|c|c|c|}
\hline & \multirow[b]{2}{*}{$M$} & \multirow[b]{2}{*}{ D.t. } & \multicolumn{5}{|c|}{ \% escala satisfacción } & Habitantes & \multirow{2}{*}{$\begin{array}{l}\text { Edad } \\
X 2\end{array}$} \\
\hline & & & 1 & 2 & 3 & 4 & 5 & $X 2$ & \\
\hline Iluminación & 4.55 & .831 & 1.6 & 1.6 & 8.1 & 18.0 & 70.8 & $14.15^{* *}$ & $8.23^{*}$ \\
\hline Limpieza & 4.03 & 1.222 & 5.9 & 7.9 & 14.4 & 21.3 & 50.5 & $16.19^{* *}$ & 7.20 \\
\hline Dimensiones del lugar & 4.16 & 1.205 & 5.7 & 6.6 & 12.0 & 17.4 & 58.4 & $15.61^{* *}$ & 5.94 \\
\hline Accesibilidad & 4.28 & 1.083 & 3.6 & 5.2 & 11.1 & 19.7 & 60.3 & $16.32^{* *}$ & 7.69 \\
\hline Ventilación & 4.43 & .918 & 1.7 & 2.8 & 11.3 & 18.8 & 65.4 & $15.61^{* *}$ & $17.56^{* *}$ \\
\hline Satisfacción con el horario & 4.54 & .822 & 1.5 & 1.6 & 7.5 & 19.6 & 69.7 & $33.83^{* *}$ & 4.17 \\
\hline Satisfacción con el tiempo de clase & 4.73 & .573 & .4 & .5 & 2.8 & 18.4 & 77.9 & $14.40^{* *}$ & 1.18 \\
\hline Satisfacción con número de horas/semana & 4.02 & 1.226 & 6.8 & 5.5 & 16.8 & 20.8 & 50.0 & 5.71 & 5.20 \\
\hline Motivación sesiones & 4.68 & .619 & .7 & .1 & 3.5 & 21.2 & 74.4 & 4.79 & 1.32 \\
\hline Intensidad sesiones & 4.64 & .652 & .4 & .8 & 4.7 & 22.6 & 71.5 & $30.50^{* *}$ & 6.41 \\
\hline Adecuación del material deportivo & 4.64 & .729 & .9 & 1.4 & 5.2 & 17.9 & 74.5 & $13.71^{* *}$ & 4.17 \\
\hline Adecuación de la duración de la clase & 4.13 & 1.093 & 3.7 & 4.8 & 17.7 & 22.8 & 51.1 & 4.43 & 7.30 \\
\hline
\end{tabular}

${ }^{*} \mathrm{p}<.05 ;{ }^{*} \mathrm{p}<.01 ; 1=$ Nada satisfecha $-5=$ Muy satisfecha

Influencia del precio mensual de la actividad en la satisfacción

El coste mensual de la actividad para las usuarias era en su mayoría gratuito, $82.7 \%$. De los que tuvieron que abonar la actividad, un $12 \%$ paga menos de $10 €$, el $3 \%$ entre $10-20 €$ y el 2.4\% más de 20€. Por último, se analizó si el hecho de pagar podía originar diferencias significativas en los ítems estudiados, encontrando que los que pagan están menos satisfechos con el número de horas semana, con la intensidad de las sesiones y con la adecuación de las instalaciones (Tabla 6).

Tabla 6. Análisis diferencial de los ítems sobre satisfacción en función de pagar por la actividad.

\begin{tabular}{lllll}
\hline & \multicolumn{4}{l}{ Diferencias Gratis / Coste Económico } \\
\hline Iluminación & $\mathrm{U}$ & $p$ & Gratis & Coste económico \\
Limpieza & 184128.00 & .993 & 802.04 & 801.83 \\
Dimensiones del lugar & 177403.50 & .288 & 807.71 & 777.64 \\
Accesibilidad & 179370.50 & .429 & 806.23 & 784.72 \\
Ventilación & 175458.00 & .157 & 795.42 & 833.36 \\
Satisfacción con el horario & 183530.00 & .913 & 802.49 & 799.68 \\
Satisfacción con el tiempo de clase & 177490.50 & .229 & 807.65 & 777.96 \\
Satisfacción con número de horas/semana & 179013.50 & .296 & 798.50 & 821.57 \\
\hline Motivación sesiones & 168347.50 & $.014^{*}$ & 814.54 & 745.07 \\
Intensidad sesiones & 180661.00 & .494 & 805.25 & 789.36 \\
Adecuación del material deportivo & 164952.00 & $.000^{* *}$ & 817.10 & 732.85 \\
Adecuación de la duración de la clase & 168288.00 & .305 & 768.01 & 796.04 \\
Horarios de las actividades & 176740.50 & .204 & 806.61 & 777.05 \\
Profesionales que imparten la actividad & 170306.50 & .062 & 811.06 & 755.55 \\
\hline
\end{tabular}

${ }^{*} \mathrm{p}<.05 ;{ }^{* *} \mathrm{p}<.01$

Por otro lado, se les preguntó por qué motivos abandonarían la práctica de AF, para ello se empleo una escala Likert de 5 pasos ( $1=$ Nada de acuerdo $-5=$ totalmente de acuerdo (Tabla 7). La falta de tiempo fue el motivo que más alto puntuó por el que abandonarían la práctica. En este mismo motivo se encon- traron diferencias significativas en función de la edad ( $p<.01)$. Así mismo, se pudo comprobar que los que pagaban una cantidad por la actividad estaban más de acuerdo por abandonar por falta de atención del monitor, falta de variedad de las actividades y falta de mantenimiento de las instalaciones. 
Tabla 7. Descriptivos y análisis diferencial a través de la h de la prueba Kruskal Wallis en función de la edad de los motivos de abandono.

\begin{tabular}{|c|c|c|c|c|c|c|c|c|c|c|c|}
\hline & & & & & & & & \multirow[t]{2}{*}{ Edad } & \multicolumn{3}{|c|}{ Gratis / Coste económico } \\
\hline & & & & & & & & & & \multicolumn{2}{|c|}{ Rangos promedio } \\
\hline & $\mathrm{M}$ & $\mathrm{Dt}$ & 1 & 2 & 3 & 4 & 5 & $\mathrm{X} 2$ & $\mathrm{U}$ & Gratis & Coste \\
\hline $\begin{array}{l}\text { Porfalta de } \\
\text { tiempo }\end{array}$ & 3.00 & 1.714 & 34.5 & 8.7 & 13.5 & 8.7 & 34.6 & $15.960^{* *}$ & 170843,50 & 780.32 & 820.96 \\
\hline $\begin{array}{l}\text { Malestar con los } \\
\text { compañeros }\end{array}$ & 2.06 & 1.292 & 52.1 & 12.3 & 19.9 & 9.3 & 6.4 & 1.911 & 161431,50 & 721.57 & 723.81 \\
\hline $\begin{array}{l}\text { Por falta de aten- } \\
\text { ción del monitor }\end{array}$ & 2.55 & 1.509 & 41.3 & 8.0 & 20.2 & 15.7 & 14.8 & 1.188 & $149034,00^{*}$ & 710.93 & 768.41 \\
\hline $\begin{array}{l}\text { Por falta de } \\
\text { variedad de } \\
\text { actividades }\end{array}$ & 2.47 & 1.411 & 38.9 & 12.7 & 22.7 & 14.6 & 11.2 & 2.337 & $146126,50^{* *}$ & 708.04 & 777.87 \\
\hline $\begin{array}{l}\text { Por falta de } \\
\text { mantenimiento de } \\
\text { instalaciones }\end{array}$ & 2.62 & 1.476 & 34.3 & 14.7 & 22.7 & 10.9 & 17.3 & 1.019 & $142466,50^{* *}$ & 703.32 & 787.03 \\
\hline
\end{tabular}

${ }^{*} p<.05 ;{ }^{* *} p<.01 ; 1=$ Totalmente en desacuerdo -5 = Totalmente desacuerdo.

A través de las pruebas post hoc realizadas a través del modelo T3 de Dunnett se pudo comprobar que había diferencias significativas entre las mujeres con menos de 70 años y las mujeres entre 70 y 79 ańos.

\section{Discusión}

Las localidades en las que más mujeres participan en las clases de actividad física de mantenimiento, actividad incluida en el Programa de Dinamización Deportiva, son las localidades menores de 5000 habitantes. En estas localidades, los programas de AF mantenimiento en el ámbito privado, gimnasios y salas de fitness, suelen ser escasos. La permanencia en estos programas de gimnasia de mantenimiento es alta, en su mayoría superior a 3 años y en línea con Meredith (1988), Quenneville y Sidney, (1992), Mahoney (1993) y Morrow y Gill (1995).

El estudio confirma que el principal motivo de las mujeres mayores para participar en los programas de AF de mantenimiento es la salud en línea con Moreno y Marín de Oliveira (2003), y que este motivo es la principal razón para mantener la participación en este programa. Se evidencia por tanto la relación entre la actividad física y la mejora de la salud en esta población en línea con los resultados de Pastón et al. (2011).

La recreación y relación social son otros motivos que manifiestan las encuestadas, aunque muy alejados de los de salud, demostrando el papel socializador esta actividad (Prevc y Tepic, 2009; Sterbova, Hrubá, Harvanova, Elfmark y Otipkova; 2009) . En función de la edad, no se aprecian diferencias significativas entre los grupos de edad estudiados, siendo en más del 75\% de cada grupo la salud, el principal motivo para inscribirse en un el programa de AF. No obstante, los residuos tipificados corregidos indican que entre los
70-74 años hay más casos de los esperados de personas que se inscriben para relacionase con gente mientras que entre los 65-59 hay menos. Esta misma tendencia se mantiene en los motivos de adherencia a la práctica, una vez que ya han participado en el programa. Ello confirma las conclusiones de numerosas investigaciones que encontraron en la salud el principal motivo para practicar AF (García, et al., 1996; Pavón y Moreno, 2008), sobre todo en la práctica de AF de mantenimiento (Cabañas, et al., 2004; Moreno, Rodríguez y Martínez, 1997).

Se ha podido comprobar también que las mujeres de más edad manifiestan una mayor valoración a la mejora de sus relaciones sociales a partir del programa de AF de mantenimiento en línea con diversos autores (Cabañas, Martínez y Del Riego, 2004; Ruíz et al., 2007; Prevc y Tepic, 2009; Sterbova, Hrubá, Harvanova, Elfmark y Otipkova; 2009), que manifiestan que con la edad la motivación hacia la salud aumenta así como la necesidad de relaciones sociales.

Sin establecer ninguna relación de causalidad, dada la naturaleza de los datos, los resultados muestran una tendencia en estos programas de AF regular de mantenimiento hacia una mayor percepción de mejora en diversos ámbitos implicados con la salud, física, psicológica y social, según se aumenta la edad (Janisse, Nedd, Escamilla y Nies, 2004; Olmedilla, Ortega y Madrid, 2008; Stathi, Fox, y McKenna, 2002; McAuley, Konopack, Motl, Rosengren y Morris, 2006; White, Wojcicki y McAuley; 2009; Umstattd, Wilcox y Dowda, 2011).

De igual modo, la edad de las participantes y la composición heterogénea de los grupos en las clases, pueden ser razones para que la percepción del componente físico no tenga una importancia destacable, debido a que las clases se desarrollan con niveles de intensidad adecuados por los dinamiza- 
dores (Blázquez, Feu y Ortiz 2010) para garantizar un seguimiento de todas las participantes y teniendo como referencia a las de menor condición física.

Los resultados obtenidos en cuanto al número de habitantes y el grado de satisfacción de las diferentes variables señalarían una cierta homogeneidad en la satisfacción de las usuarias de la AF de mantenimiento desarrolladas a través del Programa de dinamización deportiva respecto al número de horas a la semana, adecuación del material e instalaciones, así como la motivación de las sesiones. Sin embargo, las diferencias respecto a la satisfacción que presentan las usuarias se centran en relación a la instalación (iluminación, limpieza, dimensiones del lugar, accesibilidad y ventilación) y en cuanto a la organización (horario, duración, intensidad y tiempo efectivo de práctica en la sesión), factores propiamente determinados por los recursos municipales y la planificación de la actividad por parte de los dinamizadores deportivos.

Destacar que las mayores diferencias en relación al número de habitantes y la satisfacción de las usuarias se presentan principalmente en la intensidad de las sesiones y el horario de la actividad, aspectos que estarían muy relacionados con la compatibilidad horaria del dinamizador para desarrollar su labor en las diferentes que gestiona y donde imparte tanto esta actividad físico deportiva como otras.

La explicación de las diferencias significativas en función de los rangos de edad respecto a la ventilación e iluminación de las instalaciones, podrían entenderse teniendo en cuenta los estudios sobre la agudeza visual de Baker (1987); y olfativa de Vroon, (1999) y que no solo evidencian diferencias perceptivas visuales y olfativas según el sexo de las personas sino que en edades avanzadas, es probable que su nivel de agudeza pueda diferir también de unas personas a otras. De esta forma, la declinación visual y olfativa que experimenta la mujer con la edad podría contribuir a una menor percepción que llevaría a una menor exigencia y por tanto, a una mayor satisfacción siguiendo los postulados de Simona (1969).

Para entender mejor los resultados referidos a la variable precio mensual y el nivel de satisfacción de las usuarias, es importante tener en cuenta los postulados de Nagel (1995) donde el valor de un servicio no sólo está determinado por su cuantía económica sino también por la precisión con la que los compradores perciben dicho valor, y por la importancia que otorgan a conseguir lo máximo posible con su dinero o esfuerzo. En este sentido, los resultados ofrecidos parecen mostrar los aspectos que con mayor precisión valoran y a los que intentan lograr el máximo rendimiento por su aportación económica. Así la cuantía del precio a abonar en el servicio deportivo provoca una mayor atención de las usuarias sobre dos aspectos organizativos de la actividad: número de horas semanales e intensidad de la sesión, y sobre la adecuación de la instalación. De hecho, las mayores diferencias significativas respecto a la variable precio (pagar o no pagar), se centran en la adecuación de las instalaciones y el número de horas a la semana de práctica.

Esta misma variable de cuantía del precio abonado por la actividad relacionándola con los motivos de abandono, parece seguir poniendo el acento en el mantenimiento de la instalación pero también en el profesional que la imparte, siendo su falta de atención y variedad en la actividad argumentos de abandono claves cuanto mayor es el precio que abona la usuaria.

Por último, las diferencias significativas que se presentan con la edad (a partir de los 70 años) en la opinión sobre los motivos de abandono derivados de la falta de tiempo podrían explicarse teniendo en cuenta que a partir esta edad, la disponibilidad de tiempo útil puede variar dependiendo de factores tales como el propio estado de salud o su rol familiar (cuidado de familiares, nietos, etc.).

\section{Conclusiones}

La salud es el principal motivo de las mujeres mayores para la inscripción y en la permanencia en la AF de mantenimiento en los programas de dinamización deportiva en el ámbito municipal rural de Extremadura. Otros motivos como la relación con los demás, la recreación o la preparación para el deporte son elegidos minoritariamente como motivos para practicar en un programa de AF de mantenimiento. Las mujeres perciben subjetivamente una mejora en distintos elementos vinculados a la salud en sus tres niveles: físico, bienestar consigo mismas y en las relaciones sociales.

Las usuarias que más pagan por este tipo de actividades están más satisfechas con la relación calidad/precio del servicio. Por otro lado, se observa que las usuarias que más pagan presentan una menor satisfacción con el número de horas semana, la intensidad de las sesiones, y la adecuación de las instalaciones.

La falta de tiempo fue el motivo que más alto puntuó por el que abandonarían la práctica, encontrándose diferencias significativas en función de la edad y comprobando que las que pagan una cantidad por la actividad están más de acuerdo en abandonarla por falta de atención del monitor, falta de variedad de las actividades y falta de mantenimiento de las instalaciones.

\section{Referencias}

Águila, C., Sicilia, A. Muyor, J.M. y Orta, A. (2009). Cultura posmoderna y perfiles de práctica en los centros deportivos municipales. Revista Internacional de Medicina y Ciencias de la Actividad Física y el Deporte,
9(33) 81-95.

Annesi, J., Unruh, J.L., Marti, C.N., Gorjala, S. y Tennant, G. (2011). Effects of the coach approach intervention on adherence to exercise in 
obese women: assessing mediation of social cognitive theory factors. Research quarterly for exercise and sport, 82 (1), 99-108.

Baker, E. (1987). Reducing bias and inefficiency in the selection algorithms, Proceedings of the Second International Conference on Genetic Algorithms, Grafensttete, 1, 14-21.

Bitner, M.J. (1990). Evaluating service encounters: the effects of physical surroundings and employee responses. Journal of Marketing, 54, 69-82.

Blázquez, A., Feu, S. y Ortiz, E. (2010). Aprendizaje Cooperativo a través de las TICS: una experiencia en dinamización deportiva. Mérida: Escuela de Administración Pública de Extremadura.

Bolton, R.N., y Drew, J.H. (1991). A longitudinal analysis of the impact of service changes on customer attitudes. Journal of Marketing, 55, 1-9.

Boulding, W., Kalra, A., Staelin, R., y Zeithml, V.A. (1993). A dynamic process model of service quality: from expectations to behavioral intentions. Journal of Marketing Research, 30, 7-27.

Cabañas, R., Martínez, P. y Del Riego, M.L. (2004). Análisis de la motivación de las mujeres para la práctica de actividades físicas según la edad. Educación Física y Deportes, 72. Consultado 10/07/2010 desde http:// www.efdeportes.com/efd72/mujeres.htm.

Calabuig, F., Molina, N. y Nuñez, J. (2012). Una aplicación inicial del modelo tridimensial de calidad en centros deportivos privados. E-balonmano: Revista de Ciencias del Deporte, 8 (1), 67-81.

Cronin, J. Jr. Y Taylor, S. (1992). Measuring servce quality: a re-examination and extension. Journal of Marketing, 56, 55-68.

Choi, K.S., Cho, W.H., Lee, S., Lee, H., y Kim, C. (2002). The relationship among quality, value, satisfaction and behavioral intention in health care provider choice: a South Korean study. Journal of Business Research, 57(38), 1-9.

Fornell, C. (1996). The American Customer Satisfaction Index: Nature, Purpose and Findings. Journal of Marketing, 60, 7-18.

García, M.E., Hernández, A.I., Ońa, A., Godoy, J.F. y Rebollo, S. (2001). La práctica física de tiempo libre en la mujer. Revista Motricidad 7, 145 186.

Graupera, J.L., Martínez Del Castillo, J. y Martín, B. (2003). Factores motivacionales, actitudes y hábitos de práctica de actividad física en las mujeres mayores. Serie ICD de Investigación en Ciencias del Deporte, 35 , 181-222.

Janisse, H., Nedd, D., Escamilla, S. y Nies, M. (2004). Physical activity, social support, and family structure as determinants of mood among European- American and African-American women. Women Health, 39(1), 101-116.

Johnson, J.W., y Gustavson, E. (2000). Linking employee perceptions of service climate to customers satisfaction. Personnel Psychology, 49, 831851.

Macías, V. y Moya, M. (2002). Género y Deporte. La influencia de las variables psicosociales sobre la práctica deportiva de jóvenes de ambos sexos. Revista de Psicología Social, 17(2), 129-148.

Magretta, J. (2002). What Management is. How it works and why it's everyone's business. New York: The Freepress.

Mahoney, C. (1993). Health Related Exercise in Northern Ireland. The Bulletin of Physical Education, 29(2), 21-24.

Mármol, A., Orquín Castrillón, F. J. y Sainz de Baranda, P. (2010). La infraestructura y el equipamiento, la prescripción del ejercicio y los servicios ofertados como índices de calidad de los centros fitness de Murcia. Cuadernos de Psicología del Deporte, 10,(Suple), 85-91.

Martin, S. L., Kirkner, G. J., Mayo, K., Matthews, C. E., Durstine, J. L., \& Hebert, J. R. (2005). Urban, rural, and regional variations in physical activity. Journal of Rural Health, 21(3), 239-244.

Martínez, J.A. y Martínez, L. (2009). ¿Las actitudes hacia el servicio deportivo varían según el grado de experiencia del consumidor?. Motricidad. European Journal of Human Movement, 22, 133-146.

Martínez-Tur, V., Peiró, J. y Ramos, J. (1995). Efecto modulador de los aspectos sociodemográficos en la predicción de la práctica deportiva y uso de instalaciones deportivas. Anales de Psicología, 11(1), 77-96.

McAuley, E., Konopack, J.F., Motl, R.W., Rosengren, K. y Morris, K.S. (2006). Physical activity and quality of life in older adults: Influence of health status and self-efficacy. Annals of Behavioral Medicina, 31 (1), 99-103.

Meredith, M. D. (1988). Activity or fitness: Is the process or the product more important for public health? Quest, 40, 180-186.

Montero, I. y León, O.G. (2007). Guía para nombrar los estudios de investigación en Psicología. International Journal of Clinical and Health Psychology, 7, 847-862.

Morales, V., Hernández, A. y Blanco, A. (2005). Evaluación de la calidad en programas de actividad física. Psicothema, 17(2), 292-298.

Moreno, J.A. y Gutiérrez, M. (1998). Bases metodológicas para el aprendizaje de las actividades acuáticas educativas. Barcelona: INDE.

Moreno J.A., y Marín de Oliveira, L.M. (2003). Análisis de los motivos de práctica entre usuarios de programas tradicionales y de fitness. Actas del Congreso Internacional de Actividades Acuáticas (pp. 26-28). Murcia: Universidad de Murcia.

Moreno, J.A., Rodríguez, P.L. y Martínez, A. (1997). Estudio de los motivos de la práctica físico-deportiva como elemento de ajuste entre la oferta y la demanda. Perspectivas, 2, 45-55.

Morrow, J.R. y Gill, D.L. (1995). Physical Activity, Fitness and Health: Introduction. Quest, 47(3), 201-202.

Nagel, T. (1995). The strategy and Tactics of Pricing, Prentice Hall, 2 Ed., Englewood Cliffs, NJ.

Nuviala, A., Tamayo, J. A., Iranzo, J. y Falcón, D. (2008). Creación, diseño, validación y puesta en práctica de un instrumento de medición de la satisfacción de usuarios de organizaciones que prestan servicios deportivos. Retos. Nuevas perspectivas de Educación Física, Deporte y Recreación, 14, $10-16$.

Oliver, R.L. (1993). A Conceptual Model of Service Quality and Service Satisfaction: Compatible Goals. Different Concepts. Advances in Services Marketing and Management, 2, 65-85.

Olmedilla, A., Ortega, E. y Madrid, J. (2008). Variables sociodemográficas, ejercicio físico, ansiedad y depresión en mujeres: un estudio correlacional. Revista Internacional de Medicina y Ciencias de la Actividad Física y el Deporte, 8 (31), 224-243.

Paxton, R.J., Motl, R.W., Aylward, A. y Nigg, C.R. (2011). Physical Activity and Quality of Life-The Complementary Influencia of Self-Efficacy for Physical Activity and Mental Health Dificulties. International Journal of Behavioral Medicine, 17 (4), 255-263.

Pavón, A. y Moreno, J.A. (2008). Actitud de los universitarios ante la práctica físico deportiva: diferencias por géneros. Revista de Psicología del Deporte, 17(1), 7-23

Prevc, P. y Tepic, M.D. (2009). Age indentity, social influence and socialization through physical activity in elderly people living in a nursing home. Collegioum Antropologicum, 33 (4), 1107-1114.

Quenneville, M.J.J. y Sidney, K. (1992). Fitness Testing and Counselling in Health Promotion. Canadian Journal of Sport Science, 17(3), 194-198.

Reis, J.P., Bowles, H.R., Ainsworth, B.E., Dubose, K.D., Smith, S., y Laditka, J.N. (2004). Nonoccupational physical activity by degree of urbanization and US geographic region. Medicine and Science in Sports and Exercise, 36(12), 2093-2098.

Rodríguez-Romo, G., Boned-Pascual, C., y Garrido-Muñoz, M. (2009). Motivos y barreras para hacer ejercicio y practicar deportes en Madrid. Revista Panamericana de Salud Publica, 26(3), 244-254.

Ruíz, F., García, M.E., Díaz, A. (2007). Análisis de las motivaciones de práctica de actividad física y de abandono deportivo en la Ciudad de La Habana (Cuba). Anales de Psicología, 23(1), 152-166.

Sen, S., y Johnson, E, (1997). Mere-Possession effects without possession in Consumer Choice, Journal of Consumer Research, 24, 232-247.

Sterbova, D., Hruba, R., Harvanova, J., Elfmark, M. y Otipkova, D. (2008). Factors of adherence to physical activity in women aged 40-65 
years. Ceskoslovenska Psychologie, 52 (4), 378-387.

Simone, S. (1969). El carácter de las mujeres. Barcelona: Herder.

Smith, A.L. (1999). Perceptions of peer relationships and physical activity participation in early adolescence. Journal of Sport \& Exercise Psychology, 21, 329-350.

Spreng, R.A., y Mackoy, R.D. (1996). An empirical examination of a model of perceived service quality and satisfaction. Journal of Retailing, 72(2), 201-214.

Stathi, A., Fox, K. y McKenna, J. (2002). Physical Activity and dimensions of subjective well-being in older adults. Journal of aging and physical activity, 10, 76-92.

Tay J.B., Kelleher C.C., Hope A., Barry, M., Gabhainn, S.N., y Sixsmith, J. (2004). Influence of sociodemographic and neighbourhood factors on self rated health and quality of life in rural communities: findings from the Agriproject in the Republic of Ireland. Journal of Epidemiology and Community Health. 58(11), 904-911.

Taylor, S.A. Y Baker T.L. (1994). An assessment of the relationship between service quality and customer satisfaction in the formation of consumers' purchase intentions. Journal o Retailing, 70(2), 163-178.

Tsitskari. E., Tsiotras, D. y Tsiotras, G. (2006). Measuring service quality in sport services. Total Quality Management \& Business Excellence, 17(5)
623-631

Umstattd, M.R., Wilcox, S. y Dowda, M. (2011). Predictors of change in satisfaction with body appearance and body function in mid-life and older adults: active for life. Annals of behavioural medicine, 41 (3), 342 352.

Vroon, P. (1999). La seducción secreta. Psicología del olfato. Barcelona: Tusquets Editores.

Weiss, M.R., y Ebbeck, V. (1996). Self-esteem and perceptions of competence in youth sport: Theory, research, and enhancement strategies. En O. Bar-Or (Ed.) The encyclopaedia of sports medicine, Volume VI: The child and adolescent athlete (pp. 364-382). Oxford: Blackwell Science Ltd.

White, M., Wojcicki, T.R. y McAuley, E. (2009). Physical activity and quality of life in community dwelling older adults. Health and Quality of life outcomes, 7 (1).1-10

Wicker, P., Breuer, C., y Pawlowski, T. (2009). Promoting sport for all to age-specific target groups - The impact of sport infrastructure. European Journal of Marketing, 9(2), 103-118.

Woodside, A.G.; Frey, L.L., y Daly, R.T. (1989). Linking service quality, customer satisfaction and behavioral intention. Journal of Health Care Marketing, 9, 5-17.

\section{Apéndice}

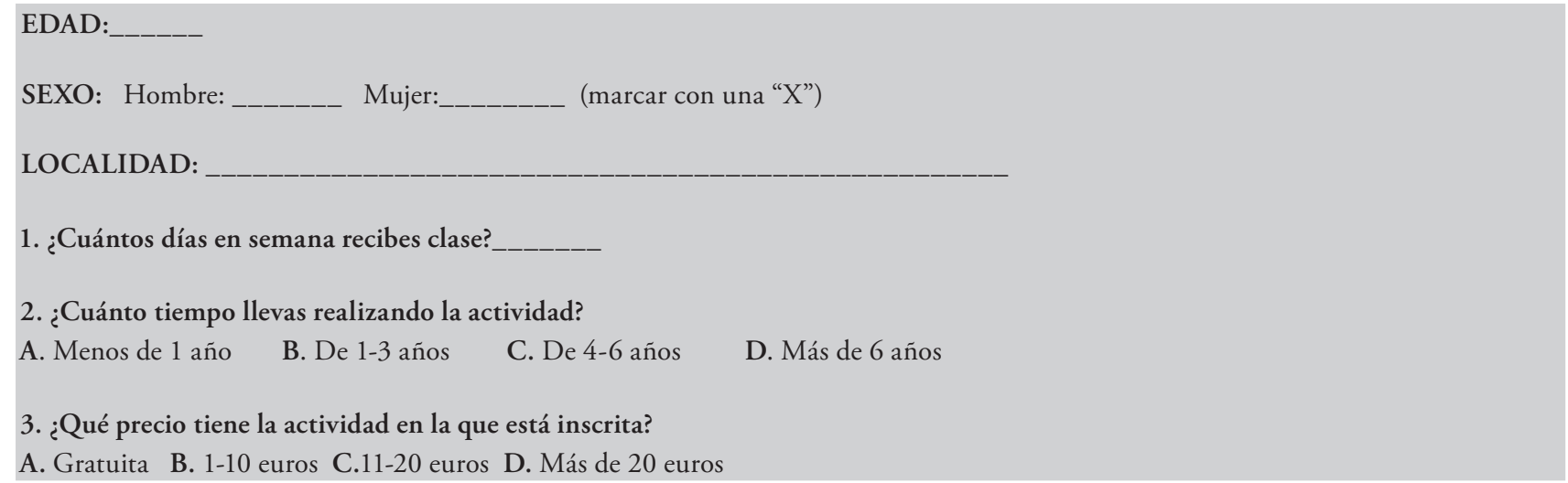

\section{BLOQUE: INGRESO EN LA ACTIVIDAD}

4. ¿POR QUÉ MOTIVO PRINCIPAL DECIDISTE INSCRIBIRTE? (Rodear con un círculo 1 OPCION)
A. Estética
B. Salud
C. Recreación
D. Encontrarse con gente
E. Preparación física

\section{BLOQUE: SATISFACCIÓN CON EL SERVICIO}

\section{INDIQUE SU SATISFACCIÓN CON LA ADECUACIÓN DE LAS INSTALACIO- NES A TRAVÉS DE LOS SIGUIENTES ÍTEMS (Rodear con un círculo)}

Satisfecho

Nada
A. La iluminación de la sala
$\begin{array}{lllll}1 & 2 & 3 & 4 & 5\end{array}$
B. La limpieza de la sala
$\begin{array}{lllll}1 & 2 & 3 & 4 & 5\end{array}$
C. El tamaño de la sala
$\begin{array}{lllll}1 & 2 & 3 & 4 & 5\end{array}$
D. La accesibilidad a las salas
$\begin{array}{lllll}1 & 2 & 3 & 4 & 5\end{array}$ 
E. La ventilación de la sala 6. INDIQUE SU SATISFACCIÓN CON LA ADECUACIÓN DE LA ORGANIZACIÓN DE LA ACTIVIDAD A TRAVÉS DE LOS SIGUIENTES ÍTEMS (Rodear con un círculo)
A. El horario en que se desarrollan las actividades
B. Aprovechamiento del tiempo de clase.
C. Número de horas semanales dedicadas

7. INDIQUE SU SATISFACCIÓN CON LA ADECUACIÓN DE LA ACTIVIDAD A

TRAVÉS DE LOS SIGUIENTES ÍTEMS (Rodear con un círculo)

Nada Satisfecho $\begin{array}{lllll}1 & 2 & 3 & 4 & 5\end{array}$
A. Las sesiones me resultan motivantes
B. La intensidad de las sesiones es adecuada
C. El material deportivo que se utiliza es adecuado
D. La duración de la clase me parece adecuada

Nada Satisfecho

\begin{tabular}{|l|l|l|l|l|}
\hline 1 & 2 & 3 & 4 & 5 \\
\hline 1 & 2 & 3 & 4 & 5 \\
\hline 1 & 2 & 3 & 4 & 5 \\
\hline
\end{tabular}

Muy Satisfecho

\begin{tabular}{|l|l|l|l|l|}
\hline 1 & 2 & 3 & 4 & 5 \\
\hline 1 & 2 & 3 & 4 & 5 \\
\hline 1 & 2 & 3 & 4 & 5 \\
\hline 1 & 2 & 3 & 4 & 5 \\
\hline
\end{tabular}
Muy Satisfecho

BLOQUE: ADHERENCIA A LA ACTIVIDAD

8. TU PRINCIPAL RAZON PARA SEGUIR PRÁCTICANDO ESTA ACTIVIDAD ES: (Rodear con un círculo 1 OPCION)
A. Estética
B. Salud
C. Recreación
D. Encontrarse con gente E. Preparación física

9. VALORE SUS AVANCES CON LA ACTIVIDAD
A. He comprobado que mi forma física está mejorando
B. He comprobado que me encuentro mejor conmigo mismo/a
C. He comprobado que mi relación con los demás ha mejorado
10. ¿QUÉ MOTIVO PRINCIPAL TE HARÍA ABANDONAR?

Totalmente en desacuerdo

\begin{tabular}{|l|l|l|l|l|}
\hline 1 & 2 & 3 & 4 & 5 \\
\hline 1 & 2 & 3 & 4 & 5 \\
\hline 1 & 2 & 3 & 4 & 5 \\
\hline
\end{tabular}
Totalmente de acuerdo
A. Por falta de tiempo
B. Por malestar con mis compañeros/as
C. Porque el monitor/a no me preste atención
D. Por la falta de variedad en las actividades (monotonía)
E. Por falta de mantenimiento de las instalaciones

Totalmente en desacuerdo

\begin{tabular}{|l|l|l|l|l|}
\hline 1 & 2 & 3 & 4 & 5 \\
\hline 1 & 2 & 3 & 4 & 5 \\
\hline 1 & 2 & 3 & 4 & 5 \\
\hline 1 & 2 & 3 & 4 & 5 \\
\hline 1 & 2 & 3 & 4 & 5 \\
\hline
\end{tabular}

Totalmente de acuerdo 